
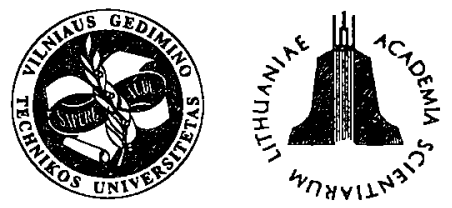

ISSN 1648-4142 TRANSPORT

http:/www.vtu.lt/english/editions

TRANSPORT - 2002, Vol XVII, No 5, 177-18I

\title{
PIPELINE ROBOTS WITH ELASTIC ELEMENTS
}

\author{
Arvydas Matuliauskas', Bronislovas Spruogis ${ }^{2}$ \\ Department of Transport Technologic Equipment, Vilnius Gediminas Technical University, \\ Plytines g. 27, LT-2040 Vilnius, Lithuania,.Tel. (22)699705; E-mail: tti@ti.vtu.lt
}

Received 200205 27; accepted 20020910

\begin{abstract}
In the article constructions of the pipeline robots with elastic elements are reviewed and the scheme of new original construction is presented. The mathematical models of a robot with one-dimensional vibration exciter with two degrees of freedom were developed and the equations of movement were formed and written. The mathematical model of the pipeline robot with circular elements is formed and its motion equations are presented.
\end{abstract}

Keywords: a pipeline robot with elastic elements, robot with ring elements, vibration exciter.

\section{Introduction}

Robots of various types and designs are widely used for effective control and repair of gas pipelines, water pipelines or communication lines [1,2]. One kind namely, robots those with elastic elements is discussed here. The object of the present project is the development of an original robot with elastic ring elements and the investigation of its movement.

\section{Designs of Robots With Elastic Elements}

Usually a robot consists of several transporting blocks connected with elastic elements [3] (Fig 1). The transporting blocks are equipped with elements that contact with the internal surface of the pipe in several points and ensure fixing of the block in the pipe. Because of a simple structure and sufficiently good fixation in the pipe robots with elastic elements consisting of rings, half-rings and similar easily deformable elements may be usable as well.

A transporting block of such robots consists of a drive, deformable elastic elements that pay a role of block fixing supports and block connecting elements. When one block is fixed, other blocks may turn and move along the fixed block. This enables the equipment to go round obstacles on a movement in the pipe.

However, some robots with transporting blocks of this type have some imperfections (such as large sizes, limited power supply possibilities) that cause limited possibilities of their application. The most frequent imperfection - such robots may be used only in straight fragments of pipelines (Fig 1) and their capability to move in sections of different diameters of pipes is limited.

So, one of newer designs providing extended possibilities of application is a robot with ring elements, con- sisting of transporting blocks connected with elastic elements (Figs 2,3). Such robots consist of rings or their elements with the ends of the latter connected with elastic link elements and with each other forming blocks. The interconnected blocks form a chain of the blocks.

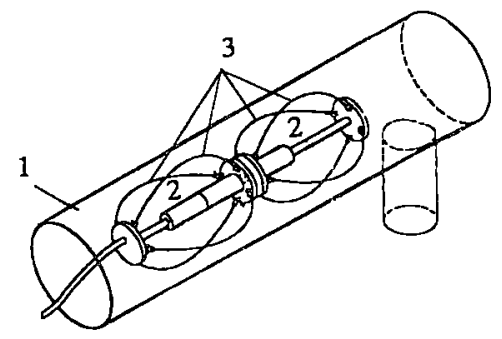

Fig 1. The scheme of a robot with ring-shaped elements: 1 - pipe, 2 - transporting blocks, 3 - elastic support elements

a)
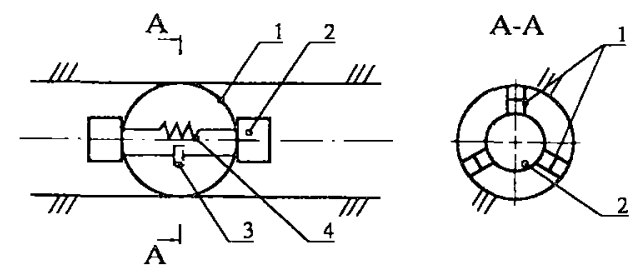

b)

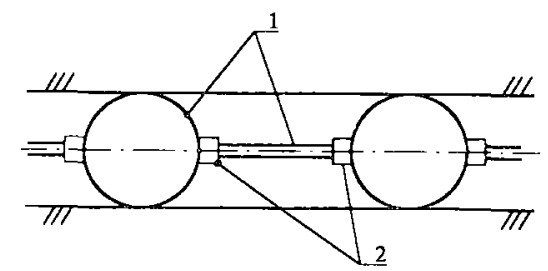

Fig 2. The schemes of the robot block with ring elements a) and its connection $b$ ):

1 -ring-shaped elastic element, 2 - the element connecting blocks and their ring elastic elements, 3 -damper, 4 - spring 

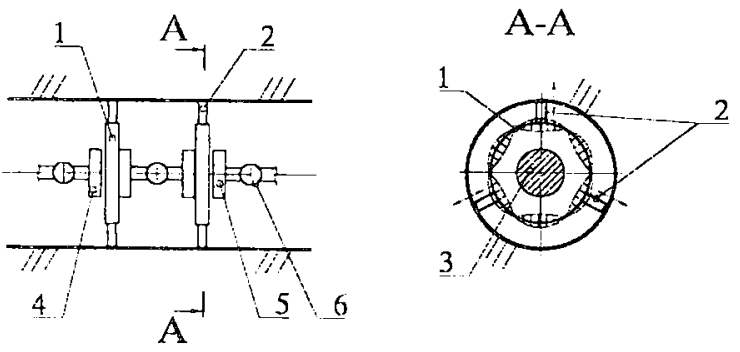

Fig 3. The scheme of a robot blocks with ring elements located along the direction of its movement:

1 - elastic ring element, 2 - supports with excitable masses,

3,5 - clectrostatic exciting elements, 4 - elastic element, 6 -hinge block jointing element

In such a way an increased capability of the robot to manoeuvre and permeability of the robot on its movement in pipes is ensured.

\section{The Mathematical Model of A Robot With Ring Ele- ments Located Perpendicularly to the Direction of its Movement}

The mathematical model of a robot is developed for the determination of its characteristics and at the beginning it may be usable in a simplified form. So, a robot with elastic ring elements situated perpendicularly to the direction of its motion (Fig 3) may be simulated by one-support dynamic model and examined as a dynamic model of onedimensional vibration exciter with two degrees of freedom (Fig 4).

The coordinates of the point $A\left(x_{0} ; y_{0}\right)$ of the excitable mass $m$ in the coordinate system $X_{0} O_{0} Y_{O}$ and the point $A(x, y)$ of the same mass in the coordinate system $X O Y$ are expressed by the following equations $[3,4]$ :

$$
\begin{aligned}
& x=z+x_{0} \cos \alpha-y_{0} \sin \alpha ; \\
& y=x_{0} \sin \alpha+y_{0} \cos \alpha \\
& x_{0}=(x-z) \cos \alpha+y_{0} \sin \alpha
\end{aligned}
$$

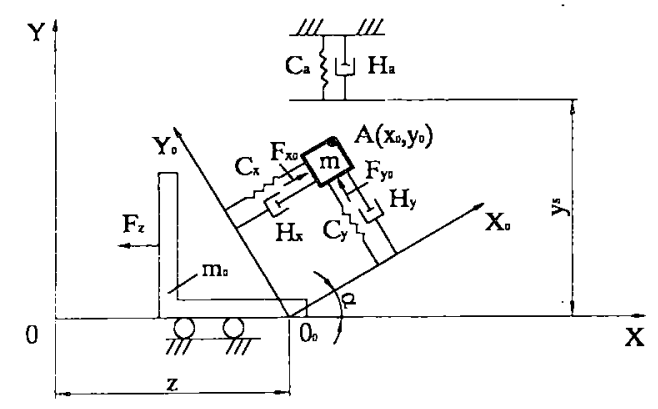

Fig 4. The scheme of the dynamic model of the system of the one-dimensional vibration exciter with two degrees of freedom

$$
y_{0}=-(x-z) \sin \alpha+y \cos \alpha
$$

The kinetic and potential energy of the system as well as its dissipative function shall be expressed as follows:

$$
\begin{aligned}
& T=0,5\left[m_{0} \dot{z}^{2}+m\left(\dot{x}^{2}+\dot{y}^{2}\right)\right] \\
& \Pi=0,5\left[c_{x} x_{0}^{2}+c_{y} y_{0}^{2}+c_{a}\left(y-y_{s}\right)^{2}\right] ; \\
& D=0,5\left[H_{x} \dot{x}_{0}^{2}+H_{y} \dot{y}_{0}^{2}+H_{u} \dot{y}^{2}\right]
\end{aligned}
$$

where $m$-mass; $x, x_{i y}, y, y_{0}$ - shifts of the robot and its mass; $y_{s}$-distance between support and the coordinate axel $X, c_{x}, c_{,}, c_{a}$ - coefficients of stiffness; $H_{a}, H_{,} H_{a}-$ coefficients of resistance of a linear shift; $f_{(p)} f_{1}$ - the coefficients of dry and liquid friction.

The mathematics expression of the dynamic model of the system shall be the following equations:

- when $y \leq y_{s}$, there is no contact with the support at the point $A$

$$
\begin{aligned}
& m \ddot{x}+c_{x}[(x-z) \cos \alpha+y \sin \alpha] \cos \alpha+ \\
& +c_{y}[(x-z) \sin \alpha-y \cos \alpha] \sin \alpha+ \\
& +H_{x}[(\dot{x}-\dot{z}) \cos \alpha+\dot{y} \sin \alpha] \cos \alpha+ \\
& +H_{y}[(\dot{x}-\dot{z}) \sin \alpha-\dot{y} \cos \alpha] \sin \alpha=F_{x} \\
& m \ddot{y}+c_{x}[(x-z) \cos \alpha+y \sin \alpha] \sin \alpha- \\
& -c_{y}[(x-z) \sin \alpha-y \cos \alpha] \cos \alpha+ \\
& +H_{x}[(\dot{x}-\dot{z}) \cos \alpha+\dot{y} \sin \alpha] \sin \alpha- \\
& -H_{y}[(\dot{x}-\dot{z}) \sin \alpha-\dot{y} \cos \alpha] \cos \alpha=F_{y} \\
& m_{0} \ddot{z}-c_{x}[(x-z) \cos \alpha+y \sin \alpha] \cos \alpha- \\
& -c_{y}[(x-z) \sin \alpha-y \cos \alpha] \sin \alpha- \\
& -H_{x}[(\dot{x}-\dot{z}) \cos \alpha+\dot{y} \sin \alpha] \cos \alpha- \\
& -H_{y}[(\dot{x}-\dot{z}) \sin \alpha-\dot{y} \cos \alpha] \sin \alpha=F_{z}
\end{aligned}
$$

- when $y \geq y_{s}$, there is a contact with the support at the point $A$ and the following forces appear:

Along the axis $O Y$ :

$$
F_{s o}=H_{a} \dot{y}+c_{a}\left(y-y_{s}\right) \text {. }
$$

Along the axis $0 X$ :

$$
F_{f}=f_{0} N \operatorname{sign} \dot{x}+f_{\mathrm{l}} \dot{x} .
$$

The normalized force of pressure:

$$
N=F_{\text {so }}
$$


$m \ddot{x}+c_{x}[(x-z) \cos \alpha+y \sin \alpha] \cos \alpha+$

$+c_{y}[(x-z) \sin \alpha-y \cos \alpha] \sin \alpha+$

$+H_{x}[(\dot{x}-\dot{z}) \cos \alpha+\dot{y} \sin \alpha] \cos \alpha+$

$+H_{y}[(\dot{x}-\dot{z}) \sin \alpha-\dot{y} \cos \alpha] \sin \alpha+F_{f}=F_{x}$;

$m \ddot{y}+c_{x}[(x-z) \cos \alpha+y \sin \alpha] \sin \alpha-$

$+c_{y}[(x-z) \sin \alpha-y \cos \alpha] \cos \alpha+$

$+H_{x}[(\dot{x}-\dot{z}) \cos \alpha+\dot{y} \sin \alpha] \sin \alpha-$

$-H_{y}[(\dot{x}-\dot{z}) \sin \alpha-\dot{y} \cos \alpha] \cos \alpha+F_{\text {so }}=F_{y}$;

$m_{0} \dot{z}-c_{x}[(x-z) \cos \alpha+y \sin \alpha] \cos \alpha-$

$-c_{y}[(x-z) \sin \alpha-y \cos \alpha] \sin \alpha-$

$-H_{x}[(\dot{x}-\dot{z}) \cos \alpha+\dot{y} \sin \alpha] \cos \alpha-$

$-H_{y}[(\dot{x}-\dot{z}) \sin \alpha-\dot{y} \cos \alpha] \sin \alpha=F_{z}$.

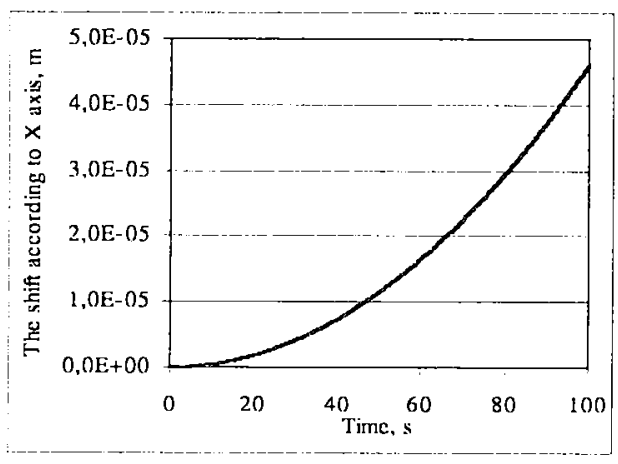

Fig 5. The shifts of the exciter according to $X$ axis dependence upon the time

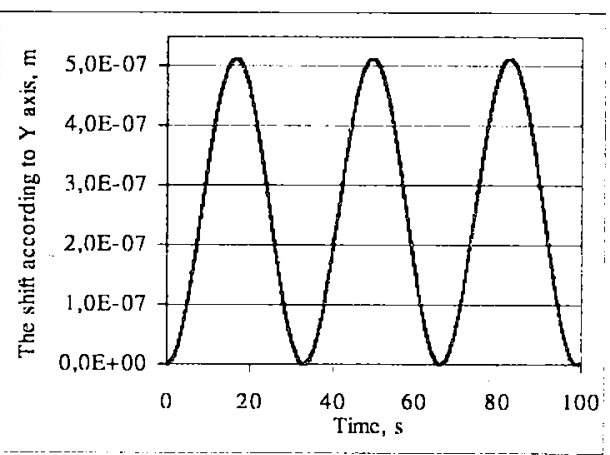

Fig 6. The shifts of the exciter according to $Y$ axis dependence upon the time
After the solution of the above-presented equations we find the characteristics of the dynamic model of the one-dimensional vibration exciter with two degrees of freedom (Figs 5, 6).

On the further analysis of the obtained solution and the optimization of the parameters of the dynamic models it was concluded that the design of the robot requires improvement. The obtained shifts of the vibration exciter were very small increasing the exciting force. So in order to ensure more effective dynamic characteristics of the robot it was decided to change its design for the one where deformable rings are deformed in the direction of the movement of the robot (Figs 2,7).

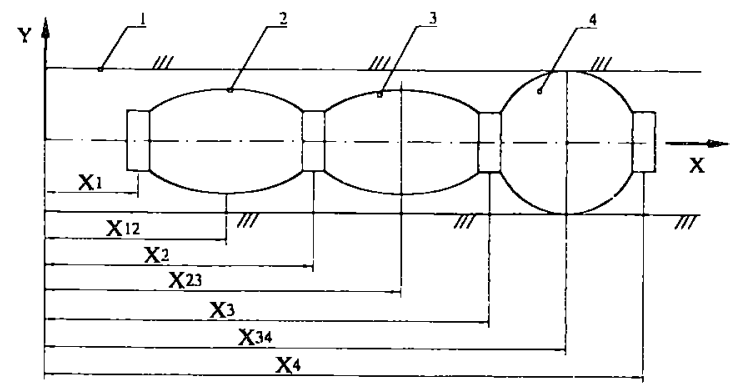

Fig 7. The scheme of a robot with ring elements

1 -pipe, 2, 3, 4-the blocks formed of elastic elements and united into the chain

\section{The Mathematical Model of a Robot with Ring Ele- ments Situated Along the Direction of its Movement}

Developing a mathematical model of a robot with elastic ring-shaped elements (Figs 2, 7) some changes are introduced to simplify the calculations. Points of a ring element may be singled out and their coordinates may be used for the description of the model or the ring elements may be replaced with solid members.

The coordinates of the points of the simplified model of a robot (Fig 8) [4] may be written as follows:

$$
\begin{aligned}
& A_{i, j+1}\left(X_{i, j+1} L_{0}+Y_{i, j+1}\right), \\
& X_{i, i+1}=\frac{1}{2}\left(X_{i}+X_{i+1}\right) ; \\
& Y_{i, i+1}=\sqrt{R^{2}-\frac{1}{4}\left(X_{i}-X_{i+1}\right)^{2}} ;
\end{aligned}
$$

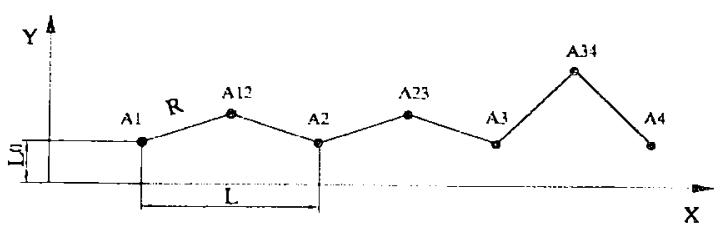

Fig 8. The simplified scheme of a robot calculation 
here $R$-the length of a section;

$$
\begin{aligned}
& \dot{X}_{i, i+1}=\frac{1}{2}\left(\dot{X}_{i}+\dot{X}_{i+1}\right) ; \\
& \dot{Y}_{i, i+1}=-\frac{X_{i}-X_{i+1}}{4 Y_{i, i+1}}\left(\dot{X}_{i}-\dot{X}_{i+1}\right) ;
\end{aligned}
$$

here $\cdot=\frac{d}{d t}$.

The kinetic energy of the system

$$
T=\frac{1}{2} m \sum_{i=1}^{q}\left(\dot{X}_{i, i+1}^{2}+\dot{Y}_{i, i+1}^{2}\right)+\frac{1}{2} m_{0} \sum_{i=1}^{q+1}\left(\dot{X}_{i}^{2}\right)
$$

here $q=1,2, \ldots n$

where

$$
\begin{aligned}
& \dot{X}_{i, i+1}^{2}+\dot{Y}_{i, i+1}^{2}=\frac{1}{4}\left(\dot{X}_{i}+\dot{X}_{i+1}\right)^{2}+ \\
& +{ }_{4}^{1} K_{i, i+1}\left(\dot{X}_{i}-\dot{X}_{i+1}\right)^{2} ; \\
& K_{i, i+1}=\frac{1}{4}\left(\frac{X_{i}-X_{i+1}}{Y_{i, i+1}}\right)^{2}=K_{i, i+1}\left(X_{i}-X_{i+1}\right) ;(24) \\
& T=\frac{m}{8}\left[\left(1+K_{12}\right)\left(\dot{X}_{1}^{2}+\dot{X}_{2}^{2}\right)^{2}+2\left(1-K_{12}\right) \dot{X}_{1} \dot{X}_{2}+\right. \\
& +\left(1+K_{23}\right)\left(\dot{X}_{2}^{2}+\dot{X}_{3}^{2}\right)^{2}+2\left(1-K_{23}\right) \dot{X}_{2} \dot{X}_{3}+ \\
& +\left(1+K_{34}\right)\left(\dot{X}_{3}^{2}+\dot{X}_{4}^{2}\right)^{2}+2\left(1-K_{34}\right) \dot{X}_{3} \dot{X}_{4}+ \\
& \left.\left(1+K_{45}\right)\left(\dot{X}_{4}^{2}+\dot{X}_{5}^{2}\right)^{2}+2\left(1-K_{45}\right) \dot{X}_{4} \dot{X}_{5}\right]+ \\
& +m_{0}\left(\dot{X}_{1}^{2}+\dot{X}_{2}^{2}+\dot{X}_{3}^{2}+\dot{X}_{4}^{2}+\dot{X}_{5}^{2}\right) .
\end{aligned}
$$

The forces of inertia are found

$$
\frac{d}{d t} \frac{\partial T}{\partial \dot{X}_{i}}-\frac{\partial T}{\partial X_{i}}=I\left(X_{i}\right)
$$

$$
\begin{aligned}
& I\left(X_{1}\right)=\frac{m}{4}\left[\left(1+K_{12}\right) \ddot{X}_{1}+\left(1-K_{12}\right) \ddot{X}_{2}+\right. \\
& \left.+\frac{1}{2}\left(K_{12}\right)_{X_{1}}^{\prime}\left(\dot{X}_{1}-\dot{X}_{2}\right)^{2}\right]+m_{0} \ddot{X}_{1} ;
\end{aligned}
$$

$I\left(X_{j}\right)=\frac{m}{4}\left[\left(1+K_{j-1 . j}\right) \ddot{X}_{j}+\left(1-K_{j-1 . j}\right) \ddot{X}_{j-1}+\left(1+K_{j, j+1}\right) \ddot{X}_{j}-\right.$

$$
\begin{gathered}
-\left(1-K_{j, j+1}\right) \ddot{X}_{j+1}-\frac{1}{2}\left(K_{j-1, j}\right)_{X_{j-1}}^{\prime}\left(\dot{X}_{j-1}-\dot{X}_{j}\right)^{2}+ \\
\left.+\frac{1}{2}\left(K_{j, j+1}\right)_{X_{j}}^{\prime}\left(\dot{X}_{j}-\dot{X}_{j+1}\right)^{2}\right]+m_{0} \ddot{X}_{j}
\end{gathered}
$$

here $j=2, \ldots, q-1$

$$
\begin{aligned}
& I\left(X_{q}\right)=\frac{m}{4}\left[\left(1+K_{q-1, q}\right) \ddot{X}_{q}+\left(1-K_{q-1, q}\right) \ddot{X}_{q-1}-\right. \\
& \left.-\frac{1}{2}\left(K_{q-1, q}\right)_{X_{q-1}}^{\prime}\left(\dot{X}_{q-1}-\dot{X}_{q}\right)^{2}\right]+m_{0} \ddot{X}_{q} .
\end{aligned}
$$

The potential energy of the system

$$
\begin{aligned}
& \Pi_{X_{1}}^{\prime}=C\left(X_{1}-X_{2}+L\right) ; \\
& \Pi_{X_{j}}^{\prime}=C\left(2 X_{j}-X_{j-1}-X_{j+1}\right) ; \\
& \Pi_{X_{q}}^{\prime}=C\left(X_{q}-X_{q-1}-L\right) .
\end{aligned}
$$

The dissipative function

$$
\begin{aligned}
& D=\frac{H}{2}\left[\left(\dot{X}_{1}-\dot{X}_{2}\right)^{2}+\left(\dot{X}_{2}-\dot{X}_{3}\right)^{2}+\right. \\
& \left.+\ldots+\left(\dot{X}_{q-1}-\dot{X}_{q}\right)^{2}\right] \\
& D_{\dot{X}_{1}}^{\prime}=H\left(\dot{X}_{1}-\dot{X}_{2}\right) ; \\
& D_{\dot{X}_{j 1}}^{\prime}=H\left(2 \dot{X}_{j}-\dot{X}_{j-1}-\dot{X}_{j+1}\right) ; \\
& D_{\dot{X}_{q}}^{\prime}=H\left(\dot{X}_{q}-\dot{X}_{q-1}\right) .
\end{aligned}
$$

The differential equations of the movement of the system

$$
\begin{aligned}
& I\left(X_{1}\right)+\Pi_{X_{1}}^{\prime}+D_{\dot{X}_{1}}^{\prime}+F_{f_{12}}=F_{12} \\
& I\left(X_{j}\right)+\Pi_{X_{j}}^{\prime}+D_{\dot{X}_{j}}^{\prime}+F_{f_{j, j-1}}+F_{f_{j, j+1}}= \\
& =-F_{j, j-1}+F_{j, j+1} ; \\
& I\left(X_{q}\right)+\Pi_{X_{q}}^{\prime}+D_{\dot{X}_{q}}^{\prime}+F_{f_{q, q-1}}=-F_{q, q-1}-F_{n} ;
\end{aligned}
$$

here $F_{n}$ - the useful resistance force.

The friction force in the point $A_{j, j+1}$ is equal to

$$
F_{f_{j, j-1}}=\frac{1}{2} N_{j, j-1} f_{0} \operatorname{sign}\left(\dot{X}_{j}+\dot{X}_{j-1}\right) ;
$$

here $N_{j, j-1}$ - the normalised force at the point $A_{j, j-1}$ acting between the immovable surface of support and the robot's ring element point $A_{j, j-1} ; f_{0}$-dry friction coefficient. At the point $A_{j}$, the external exciting force is equal to: 


$$
F_{s}=-F_{j, j-1}+F_{j, j+1}
$$

$$
\operatorname{sign}\left(\dot{X}_{j}+\dot{X}_{j-1}\right)=\left\{\begin{array}{l}
+1, \text { kai }_{j}+\dot{X}_{j-1}>0 \\
-1, \text { kaiX }_{j}+\dot{X}_{j-1}<0 \\
(-1,+1), \text { kai }_{j}+\dot{X}_{j-1}=0
\end{array}\right.
$$

When $Y_{j, j-1}=Y_{a}$, i.e. to the distance to the support, in the equations $F_{j j, j-1} \neq 0$. And when $Y_{j, j-1}<Y_{u}$, then $F_{f j . j-1}=0$.

The solution of these equations and a further analysis of the obtained results as well as the optimization of parameters of the mathematical model allow to improve the design of the robot and to develop its optimal variant.

\section{Conclusions}

Designs of robots with elastic deformable elastic ring elements are reviewed and the scheme of a new original construction is presented. Mathematical models of robots with elastic elements are developed and their dynamic equations are written.

\section{References}

1. Komori M., Suyama K. Inspection robots for gas pipelines of Tokyo Gas. Advanced Robotics, Vol 15, No 3, 2001, p $365-370$.

2. Choi H. R., Ryew S. M. Robotic system with active steering capability for internal inspection of urban gas pipelines. Mechatronics, Vol 12, Issue 5, 2002, p 713-736.

3. Kulvietis G., Mištinas V., Matuliauskas A., Ragulskis K., Spruogis B. Dynamic Parameter Investigation of Pipe Robots. Journal of Vibroengineering, No 2 (7), 2001, p 55 - 58.

4. Nimoshenko S. P., Yang D. Kh., Weaver W. Vibrations in engineering (Колебания в инженерном деле). М.: Mashinostrojenije, 1985. $472 \mathrm{p}$ (in Russian) 